\title{
ON THE DIRICHLET PROBLEM FOR MONGE-AMPÈRE TYPE EQUATIONS
}

\author{
FEIDA JIANG, NEIL S. TRUDINGER, AND XIAO-PING YANG
}

\begin{abstract}
In this paper, we prove second derivative estimates together with classical solvability for the Dirichlet problem of certain Monge-Ampère type equations under sharp hypotheses. In particular we assume that the matrix function in the augmented Hessian is regular in the sense used by Trudinger and Wang in their study of global regularity in optimal transportation [28] as well as the existence of a smooth subsolution. The latter hypothesis replaces a barrier condition also used in their work. The applications to optimal transportation and prescribed Jacobian equations are also indicated.
\end{abstract}

\section{INTRODUCTION}

This paper is concerned with existence of globally smooth solutions to the Dirichlet problem for certain Monge-Ampère type equations. These equations arise in many applications, notably in optimal transportation, geometric optics and conformal geometry. The Dirichlet problem of the Monge-Ampère type equations under consideration has the following general form

$$
\left\{\begin{aligned}
\operatorname{det}\left\{D^{2} u-A(x, D u)\right\} & =B(x, D u), & & x \in \Omega, \\
u & =\varphi(x), & & x \in \partial \Omega,
\end{aligned}\right.
$$

where $\Omega$ is a bounded domain with smooth boundary in $n$ dimensional Euclidean space $\mathbb{R}^{n}, \varphi$ is a smooth function on $\partial \Omega$. $A$ is a given $n \times n$ symmetric matrix function defined on $\Omega \times \mathbb{R}^{n}$, and $B$ is a scalar valued function defined on $\Omega \times \mathbb{R}^{n}$. We use $(x, p)$ to define points in $\Omega \times \mathbb{R}^{n}$ so that $A(x, p) \in \mathbb{R}^{n} \times \mathbb{R}^{n}$, $B(x, p) \in \mathbb{R}$. Also $D u$ and $D^{2} u$ denote the gradient vector and Hessian matrix respectively of a function $u \in C^{2}(\Omega)$. The equation in (1.1) reduces to the standard Monge-Ampère equation when $A \equiv 0$. A solution $u \in C^{2}(\Omega)$ of (1.1) is called an elliptic solution whenever $D^{2} u-A(x, D u)>0$, which implies $B>0$.

These equations have attracted significant interest in recent years; for a recent survey and the earlier history see, for example, [26]. The Heinz-Lewy example in [19] shows that there is no $C^{1}$ regularity for the equation in (1.1) without restrictions on $A$. When $A \equiv 0$, the existence of globally smooth solutions to the Dirichlet problem (1.1) for smooth uniformly convex domains was finally settled by Caffarelli, Nirenberg and Spruck [1, 2, Krylov [14] and Ivochkina [12, 13]. For application to optimal transportation with quadratic cost function, the natural boundary condition is the prescription of the gradient image $\Omega^{*}=D u(\Omega)$ which is equivalent to an oblique boundary condition for elliptic solutions. In this case local regularity for convex $\Omega^{*}$ was proved by Caffarelli [3] and the corresponding global regularity for smooth uniformly convex domains $\Omega$ and target domains $\Omega^{*}$ proved by Delanoë [6], Caffarelli [4] and Urbas [29]. The first breakthrough for general optimal transportation problems was obtained by Ma, Trudinger and Wang [18] who obtained an a priori interior second order estimate under an analytical structure condition on the matrix $A$, which they called A3, together with local regularity results under a generalized target convexity condition. These were subsequently extended to global estimates and regularity in [28] under a weak form of this condition called A3w; (see [23] for further discussion). Following this Loeper [17] found a geometric characterization enabling him to

Date: October 17, 2018.

This research was finished when the first author was visiting the Australian National University in 2011. It was supported by National Natural Science Foundation of China(No.11071119) and the Australian Research Council. 
adapt the proof of necessity of target convexity in [18] to prove that $\mathrm{A} 3 \mathrm{w}$ is necessary for regularity in optimal transportation.

In this paper we show that the condition A3w also suffices for global regularity in the Dirichlet problem not just in the optimal transportation case but also for general equations of the form (1.1). In this generality it is convenient to adopt the terminology from [23] and call the matrix function $A$ regular if

$$
A_{i j, k l}(x, p) \xi_{i} \xi_{j} \eta_{k} \eta_{l} \geqslant 0
$$

for all $(x, p) \in \Omega \times \mathbb{R}^{n}, \xi, \eta \in \mathbb{R}^{n}, \xi \perp \eta$, where $A_{i j, k l}=D_{p_{k} p_{l}}^{2} A_{i j}$.

We now formulate the main results of this paper. The first result is a global bound for second derivatives of equation (1.1) which replaces the barrier condition in [28] by the existence of a subsolution.

Theorem 1.1. Let $u \in C^{4}(\Omega) \cap C^{2}(\bar{\Omega})$ be an elliptic solution of problem (1.1) in $\Omega$, where $B \in$ $C^{2}\left(\bar{\Omega} \times \mathbb{R}^{n}\right)$, inf $B>0$ and $A \in C^{2}\left(\bar{\Omega} \times \mathbb{R}^{n}\right)$ is regular. Suppose also there exists a subsolution $\underline{u} \in C^{2}(\bar{\Omega})$ of equation (1.1). Then we have the estimate

$$
\sup _{\Omega}\left|D^{2} u\right| \leqslant C\left(1+\sup _{\partial \Omega}\left|D^{2} u\right|\right)
$$

where the constant $C$ depends on $n, A, B, \Omega, \underline{u}$ and $\sup _{\Omega}(|u|+|D u|)$.

From Theorem 1.1, we can infer a global bound for solutions of the Dirichlet problem (1.1). For this we adopt the approach from [7, 8] and instead of explicit geometric assumptions on the boundary $\partial \Omega$ as formulated for example in [23], we assume more generally the existence of a subsolution with the given boundary trace.

Theorem 1.2. In addition to the assumptions in Theorem 1.1 , suppose the subsolution $\underline{u}=\varphi$ on $\partial \Omega$ with $\varphi \in C^{4}(\bar{\Omega}), \partial \Omega \in C^{4}$. Then any elliptic solution $\left.u \in C^{4}(\Omega) \cap C^{2}(\bar{\Omega})\right)$ of the Dirichlet problem (1.1) satisfies the global a priori estimate

$$
\sup _{\Omega}\left|D^{2} u\right| \leqslant C,
$$

where the constant $C$ depends on $n, A, B, \Omega, \underline{u}$ and $\sup _{\Omega}(|u|+|D u|)$.

Existence theorems follow from Theorem 1.2 by standard methods. In general we need supplementary conditions on the matrix function $A$ to control gradients and upper bounds of solutions. These can be reduced in the optimal transportation case, where the matrix $A$ is generated by a cost function, namely a function $c \in C^{4}\left(\mathbb{R}^{n} \times \mathbb{R}^{n}\right)$ satisfying the conditions:

(A1) For each $x \in \Omega, p \in \mathbb{R}^{n}$, there exists a unique $y=Y(x, p)$ such that $D_{x} c(x, y)=p$;

$$
\operatorname{det} D_{x, y}^{2} c \neq 0 \text {, for all } x \in \Omega, y=Y(x, p), p \in \mathbb{R}^{n} \text {. }
$$

The matrix $A$ is then given by

$$
A(x, p)=D_{x}^{2} c(x, Y(x, p))
$$

We formulate here a particular theorem for the general case which also embraces the main examples in optimal transportation. For this we assume a structure condition:

$$
A(x, p) \geqslant \underset{2}{-\mu_{0}}\left(1+|p|^{2}\right) I,
$$


for all $x \in \Omega, p \in \mathbb{R}^{n}$ and some positive constant $\mu_{0}$ and that the maximum eigenvalue of $A(x, 0)$ is non-negative. The last hypothesis can be replaced by a weaker condition that there exists a bounded viscosity supersolution to equation (1.1).

We then have the following existence theorem.

Theorem 1.3. Under the assumptions in Theorem 1.2 together with the above structure conditions, there exists a unique elliptic classical solution $u \in C^{3}(\bar{\Omega})$ of the Dirichlet problem (1.1) with $u \geqslant \underline{u}$.

The paper is organized as follows: In Section 2 we prove a fundamental lemma about barriers for the linearized operator and use it to establish the global second derivative bound in Theorem 1.1. We also prove an analogous variant of the interior Pogorelev estimate of Liu and Trudinger in [16]. In Section 3, we derive the boundary estimates needed to complete the proof of Theorem 1.2. Our treatment of these boundary estimates also provides the proof of the corresponding results stated in [23] under the barrier condition introduced in [28]. In Section 4, we establish the necessary gradient and solution bounds for our existence theorems. Once we have the relevant a priori estimates, Theorem 1.3 follows immediately from the method of continuity in [11, Chapter 17]. Finally, we discuss separately the special cases of optimal transportation and prescribed Jacobian equations.

\section{Pogorelov type estimates}

In this section we prove global and interior Pogorelov type estimates for second derivatives of equation (1.1). In particular we use the regularity of the matrix function $A$ to construct a barrier function for the linearized operator from a subsolution. The resultant second derivative estimates then follow readily from the corresponding proofs in [28] and [16].

Let $F[u]=\log \left(\operatorname{det}\left(D^{2} u-A(x, D u)\right)\right)$, it is known that $F$ is a concave operator with respect to $D^{2} u-A(x, D u)$. The linearized operator of $F$ is defined by

$$
L=F^{i j}\left(D_{i j}-D_{p_{k}} A_{i j}(x, D u) D_{k}\right),
$$

where $F^{i j}=\frac{\partial F}{\partial w_{i j}}$ and $\left\{F^{i j}\right\}=\left\{w^{i j}\right\}$ denotes the inverse matrix of $\left\{w_{i j}\right\} \triangleq\left\{u_{i j}-A_{i j}(x, D u)\right\}$. Assume the function $A_{i j}(x, p) \in C^{2}\left(\bar{\Omega} \times \mathbb{R}^{n}\right), i, j=1, \cdots, n, B(x, p) \in C^{2}\left(\bar{\Omega} \times \mathbb{R}^{n}\right), \tilde{B}(x, p)=\log (B(x, p))$ and set

$$
\mathcal{L}=L-\tilde{B}_{p_{i}} D_{i}
$$

We introduce a fundamental lemma here:

Lemma 2.1. Under the above assumptions, suppose $u$ is an elliptic solution of (1.1), $\underline{u}$ is a strict elliptic subsolution of equation (1.1), if $A$ is regular, then

$$
\mathcal{L}\left(e^{K(\underline{u}-u)}\right) \geqslant \epsilon_{1} \sum_{i} F^{i i}-C,
$$

holds in $\Omega$ for positive constant $K$ sufficiently large and uniform positive constants $\epsilon_{1}, C$.

Proof. Since $\underline{u}$ is a strict subsolution of (1.1), $\underline{u}$ satisfies

$$
F[\underline{u}]=\log \left(\operatorname{det}\left(D^{2} \underline{u}-A(x, D \underline{u})\right) \geqslant \log \left(B(x, D \underline{u})+\delta_{0}\right),\right.
$$

for some $\delta_{0}>0$.

For any $x_{0} \in \Omega$, let $\underline{u}_{\epsilon}=\underline{u}-\frac{\epsilon}{2}\left|x-x_{0}\right|^{2}$. For $\epsilon$ small enough, the perturbation function $\underline{u}_{\epsilon}$ is still a strict subsolution and satisfies

$$
F\left[\underline{u}_{\epsilon}\right]=\log \left(\operatorname{det}\left(D^{2} \underline{u}_{\epsilon}-A\left(x, D \underline{u}_{\epsilon}\right)\right)\right) \geqslant \log \left(B\left(x, D \underline{u}_{\epsilon}\right)+\tau\right),
$$


for some positive constant $\tau$.

Let $v=\underline{u}-u, v_{\epsilon}=\underline{u}_{\epsilon}-u$. By calculation, we have

$$
\begin{aligned}
L v= & L\left(v_{\epsilon}\right)+L\left(\frac{\epsilon}{2}\left|x-x_{0}\right|^{2}\right) \\
= & \epsilon F^{i i}-\epsilon F^{i j} D_{p_{k}} A_{i j}(x, D u)\left(x-x_{0}\right)_{k}+F^{i j}\left\{D_{i j} v_{\epsilon}-D_{p_{k}} A_{i j}(x, D u) D_{k} v_{\epsilon}\right\} \\
= & \epsilon F^{i i}-\epsilon F^{i j} D_{p_{k}} A_{i j}(x, D u)\left(x-x_{0}\right)_{k}+F^{i j}\left\{D_{i j}\left(\underline{u}_{\epsilon}-u\right)-\left[A_{i j}\left(x, D \underline{u}_{\epsilon}\right)-A_{i j}(x, D u)\right]\right\} \\
& +F^{i j}\left\{A_{i j}\left(x, D \underline{u}_{\epsilon}\right)-A_{i j}(x, D u)-D_{p_{k}} A_{i j}(x, D u) D_{k} v_{\epsilon}\right\} .
\end{aligned}
$$

By the concavity of $F$, we have

$$
F\left[\underline{u}_{\epsilon}\right]-F[u] \leqslant F^{i j}\left\{D_{i j}\left(\underline{u}_{\epsilon}-u\right)-\left[A_{i j}\left(x, D \underline{u}_{\epsilon}\right)-A_{i j}(x, D u)\right]\right\} .
$$

By the Taylor expansion, for some $\theta \in(0,1)$, we have

$$
\begin{aligned}
& A_{i j}\left(x, D \underline{u}_{\epsilon}\right)-A_{i j}(x, D u)-D_{p_{k}} A_{i j}(x, D u) D_{k} v_{\epsilon} \\
= & D_{p_{k}} A_{i j}(x, \hat{p}) D_{k} v_{\epsilon}-D_{p_{k}} A_{i j}(x, D u) D_{k} v_{\epsilon} \\
= & \frac{\theta}{2} A_{i j, k l}(x, \bar{p}) D_{k} v_{\epsilon} D_{l} v_{\epsilon},
\end{aligned}
$$

where $\hat{p}=(1-\theta) D u+\theta D \underline{u}_{\epsilon}, \bar{p}=(1-\bar{\theta}) D u+\bar{\theta} D \underline{u}_{\epsilon}$ and $\bar{\theta} \in(0, \theta)$.

Thus, at $x=x_{0}$, we have

$$
\begin{aligned}
L v & \geqslant \epsilon F^{i i}+F\left[\underline{u}_{\epsilon}\right]-F[u]+\frac{\theta}{2} F^{i j} A_{i j, k l}(x, \bar{p}) D_{k} v D_{l} v \\
& =\epsilon F^{i i}+\frac{\theta}{2} F^{i j} A_{i j, k l}(x, \bar{p}) D_{k} v D_{l} v+\log (B(x, D \underline{u})+\tau)-\log (B(x, D u)) \\
& \geqslant \epsilon F^{i i}+\frac{\theta}{2} F^{i j} A_{i j, k l}(x, \bar{p}) D_{k} v D_{l} v-C_{1},
\end{aligned}
$$

where $C_{1}$ is a constant depends on $B, D u$, and $D \underline{u}$.

Let $\phi=e^{K v}$ with positive constant $K$ to be determined, we have

$$
\begin{aligned}
L \phi & =F^{i j}\left(D_{i j} \phi-D_{p_{k}} A_{i j}(x, D u) D_{k} \phi\right) \\
& =K e^{K v} L v+K^{2} e^{K v} F^{i j} D_{i} v D_{j} v \\
& \geqslant K e^{K v}\left\{\epsilon F^{i i}+\frac{\theta}{2} F^{i j} A_{i j, k l}(x, \bar{p}) D_{k} v D_{l} v-C_{1}+K F^{i j} D_{i} v D_{j} v\right\} .
\end{aligned}
$$

Without loss of generality, we assume that $D v=\left(D_{1} v, 0, \cdots, 0\right)$. Since $A$ is regular, we have

$$
\begin{aligned}
L \phi & \geqslant K e^{K v}\left\{\epsilon F^{i i}+\frac{\theta}{2} F^{i j} A_{i j, 11}(x, \bar{p})\left(D_{1} v\right)^{2}+K F^{11}\left(D_{1} v\right)^{2}-C_{1}\right\} \\
& \geqslant K e^{K v}\left\{\epsilon F^{i i}+\frac{\theta}{2} \sum_{i \text { or } j=1} F^{i j} A_{i j, 11}(x, \bar{p})\left(D_{1} v\right)^{2}+K F^{11}\left(D_{1} v\right)^{2}-C_{1}\right\} .
\end{aligned}
$$

Since the matrix $\left\{F^{i j}\right\}$ is positive definite, any $2 \times 2$ diagonal minor has positive determinant. This implies

$$
\left|F^{1 i}\right|^{2} \leqslant F^{11} F^{i i}
$$

Then the Cauchy inequality leads to the following inequality

$$
\left|F^{1 i}\right| \leqslant \eta F^{i i}+\frac{1}{4 \eta} F^{11}
$$

for positive constant $\eta$.

Thus, we have

$$
L \phi \geqslant K e^{K v}\left\{\epsilon F^{i i}-\frac{\theta}{2} \eta F^{i i}\left|A_{1 i, 11}(x, \bar{p})\right|\left(D_{1} v\right)^{2}-\frac{\theta}{8 \eta} F^{11}\left|A_{1 i, 11}(x, \bar{p})\right|\left(D_{1} v\right)^{2}+K F^{11}\left(D_{1} v\right)^{2}-C_{1}\right\} .
$$

Choosing $\eta$ small such that $\eta \leqslant \frac{\epsilon}{\theta \max \left\{\left|A_{1 i, 11}(x, \bar{p})\right|\left(D_{1} v\right)^{2}\right\}}$ and $K$ large such that $K \geqslant \frac{\theta \max \left|A_{1 i, 11}(x, \bar{p})\right|}{8 \eta}$, we obtain

Thus, we have

$$
L \phi \geqslant K e^{K v}\left\{\frac{\epsilon}{2} F^{i i}-C_{1}\right\}
$$

$$
\mathcal{L} \phi=L \phi-\tilde{B}_{p_{i}} D_{i} \phi \geqslant K e^{K v}\left\{\frac{\epsilon}{2} F^{i i}-C_{1}\right\}-\tilde{B}_{p_{i}} D_{i} \phi,
$$

which leads to the conclusion of Lemma 2.1 with $\epsilon_{1}=\min _{\bar{\Omega}}\left\{\frac{\epsilon}{2} K e^{K v}\right\}$ and $C=\max _{\bar{\Omega}}\left\{C_{1} K e^{K v}+\right.$ $\left.\tilde{B}_{p_{i}} D_{i} \phi\right\}$. 
Remark 2.1. The function $\phi=e^{K(\underline{u}-u)}$ is global barrier function for the linearized operator $\mathcal{L}$. The inequality $\mathcal{L}\left(e^{K(\underline{u}-u)}\right) \geqslant \epsilon_{1} \sum_{i} F^{i i}-C$ in $\Omega$ will be used to control the second order global and interior estimates in this section and the boundary estimates in the next section.

Remark 2.2. It is a fairly standard calculation that a non-strict classical subsolution of a uniformly elliptic partial differential equation can be made strict using the linearized operator and the mean value theorem. For example, if $\underline{u}$ is a non-strict subsolution, then $\underline{u}+a e^{b x_{1}}$ is a strict subsolution for small constant a and large constant b, (see [11], Chapter 3). This can also be done in a neighbourhood of the boundary, preserving boundary conditions by replacing $x_{1}$ by a defining function or more specifically the distance function. By using the mean value theorem we do not need concavity or convexity and the small constant a controls the uniform ellipticity near the subsolution. Hence we need only assume the existence of a non-strict subsolution in Lemma 2.1; the inequality (2.3) will still hold for the corresponding strict subsolution. Thus, the second order apriori estimates will also hold under the existence of a non-strict subsolution and we only need to assume a non-strict subsolution in the hypotheses for our theorems.

Remark 2.3. In the previous paper [28, to obtain the second derivative bounds, a kind of global barrier condition, called A-boundedness in [23] is assumed, namely $\Omega$ is called A-bounded with respect to $u$ if there exists a function $\varphi \in C^{2}(\bar{\Omega})$ satisfying

$$
\left[D_{i j} \varphi-D_{p_{k}} A_{i j}(\cdot, D u) D_{k} \varphi\right] \xi_{i} \xi_{j} \geqslant|\xi|^{2},
$$

in $\Omega$, for all $\xi \in \mathbb{R}^{n}$. From Lemma 2.1 it follows that $A$-boundedness can be replaced by the existence of a subsolution for second derivative estimates.

Remark 2.4. If $F$ is substituted by another concave operator $F[u]=\left(\operatorname{det}\left(D^{2} u-A(x, D u)\right)\right)^{\frac{1}{n}}$, the conclusion of this lemma still holds. The only difference is the form of $F^{i j}=\frac{\partial F}{\partial w_{i j}}$. We have $F^{i j}=$ $\frac{1}{n} f w^{i j}$ if $F[u]=\left(\operatorname{det}\left(D^{2} u-A(x, D u)\right)\right)^{\frac{1}{n}}$, while $F^{i j}=w^{i j}$ if $F[u]=\log \left(D^{2} u-A(x, D u)\right)$. Here we make a convention that $\left\{w^{i j}\right\}$ denotes the inverse matrix of $\left\{w_{i j}\right\} \triangleq\left\{u_{i j}-A_{i j}(x, D u)\right\}$.

Now from Lemma 2.1, we obtain the global Pogorelov estimate, Theorem 1.1, by appropriate adjustment to the proof of Theorem 3.1 in [28]. This is done by substituting the barrier function $\widetilde{\varphi}=\epsilon_{1}^{-1} \exp (K(\underline{u}-u))$ in place of the barrier from condition (2.5). That is we maximize the function,

$$
\exp \left\{\frac{a}{2}|D u|^{2}+b \phi\right\} w_{\xi \xi},
$$

over $\Omega$ and $|\xi|=1$, where $w_{\xi \xi}=w_{i j} \xi_{i} \xi_{j}=\left(u_{i j}-A_{i j}\right) \xi_{i} \xi_{j}, \phi=e^{K(\underline{u}-u)}$ with $K$ as in Lemma 2.1, and $a$, $b$ are positive constants to be determined. Then we arrive again at the estimates (3.11) and (3.13) in 28], but now with an additional dependence on $\underline{u}$, and Theorem 1.1 follows.

As remarked in the introduction we may similarly modify the proof of the interior Pogorelev estimate in [16] to obtain the following variant. 
Theorem 2.1. Let $u \in C^{4}(\Omega) \cap C^{0,1}(\bar{\Omega})$ be an elliptic solution of problem (1.1) in $\Omega$, where $B \in$ $C^{2}\left(\bar{\Omega} \times \mathbb{R}^{n}\right)$, inf $B>0$ and $A \in C^{2}\left(\bar{\Omega} \times \mathbb{R}^{n}\right)$ is regular. Suppose also there exists an elliptic subsolution $\underline{u} \in C^{2}(\bar{\Omega})$ of equation (1.1) and a degenerate elliptic supersolution $u_{0} \in C^{1,1}(\Omega) \cap C^{0,1}(\bar{\Omega})$ such that $u=u_{0}$ on $\partial \Omega$. Then we have for any $\Omega^{\prime} \Subset \Omega$

$$
\sup _{\Omega^{\prime}}\left|D^{2} u\right| \leqslant C,
$$

where the constant $C$ depends on $n, A, B, \Omega, \Omega^{\prime}, \underline{u}, u_{0}$, and $\sup _{\Omega}(|u|+|D u|)$.

Note that if $A(x, 0)=0$, then our estimate (2.7) agrees with the usual Pogorelov estimate [11], by taking $u_{0}=0$.

\section{Boundary ESTIMATES}

In order to complete the proof of Theorem 1.2, we need to obtain an a priori estimate for the Hessian $D^{2} u$ on the boundary $\partial \Omega$ for solutions of the Dirichlet problem (1.1). First, we note from Remark 2.2 that we can assume the subsolution $\underline{u}$ is strict provided we restrict to a neighbourhood of $\partial \Omega$. As remarked in the previous section, this can be shown by adding a function $c_{1} \exp \left(c_{2} d(x)\right)$, where $d(x)=\operatorname{dist}(x, \partial \Omega)$ denotes the distance function in $\Omega$, and $c_{1}$ and $c_{2}$ are positive constants. Accordingly, we can retain Lemma 2.1 in a neighbourhood of $\partial \Omega$, which will suffice for boundary estimates.

Next, we need to observe that the regularity of the matrix function $A$ will be preserved under coordinate changes, that is, under a coordinate change, equation (1.1) is transformed into an equation of the same form with transformed matrix $A$ satisfying (1.2) with respect to the transformed gradient variables. Specifically if we take a diffeomorphism, $y=\psi(x)$ with Jacobian matrix $J=\left\{\psi_{i j}\right\}=\left\{D_{j} \psi_{i}\right\}$, then

$$
w_{i j}(x)=\psi_{k i} \psi_{l j} D_{y_{k} y_{l}} u+D_{i}\left(\psi_{k j}\right) D_{y_{k}} u-A_{i j}\left(x, J D_{y} u\right) .
$$

The transformed matrix function $A^{\prime}$ and scalar $B^{\prime}$ are thus given by

$$
\left\{\begin{aligned}
A^{\prime}{ }_{i j}(\cdot, p) & =\psi^{i k} \psi^{j l}\left[A_{k l}\left(\psi^{-1}, J p\right)-D_{k}\left(\psi_{s l}\right) p_{s}\right] \\
B^{\prime}(\cdot, p) & =(\operatorname{det} J)^{-2} B\left(\psi^{-1}, J p\right)
\end{aligned}\right.
$$

where $\left\{\psi^{i j}\right\}=J^{-1}$. The invariance of condition (1.2) follows readily from (3.1).

Consequently, we may fix a point $x_{0} \in \partial \Omega$, which we take as the origin, and a neighbourhood $\mathcal{N}$ of $x_{0}$, such that $T=\mathcal{N} \cap \partial \Omega$ lies in the hyperplane $\left\{x_{n}=0\right\}$, and the positive $x_{n}$ axis points into $\Omega$. We then have by differentiation, $D_{\alpha \beta} u\left(x^{\prime}, 0\right)=D_{\alpha \beta} \underline{u}\left(x^{\prime}, 0\right), \alpha, \beta=1, \cdots, n-1, x^{\prime}=\left(x_{1}, \cdots, x_{n-1}\right)$, which leads to the double tangential derivative estimate $\left|D_{\alpha \beta} u(x)\right| \leqslant C, \alpha, \beta=1, \cdots, n-1$, on $T$.

We then estimate the mixed tangential-normal derivatives $D_{\alpha n} u, \alpha=1, \cdots, n-1$, on $T$ by differentiating the equation (1.1) and using the barrier in Remark 2.1. With this barrier in hand, the derivation of these estimates is similar to the special case of the standard Monge-Ampère equation, as treated for example in [11]. For completeness and later reference, we carry out the details here. First by differentiation of the equation

$$
F[u]=\log \left(\operatorname{det}\left(D^{2} u-A(x, D u)\right)\right)=\tilde{B}(x, D u),
$$

we have

$$
F^{i j}\left(u_{\alpha i j}-D_{\alpha} A_{i j}(x, D u)-D_{p_{k}} A_{i j}(x, D u) u_{\alpha k}\right)=\tilde{B}_{\alpha}(x, D u)+\tilde{B}_{p_{k}}(x, D u) u_{\alpha k}, \quad \alpha=1, \cdots, n,
$$

which leads to

$$
\mathcal{L} D_{\alpha} u=\tilde{B}_{\alpha}(x, D u)+F^{i j} D_{\alpha} A_{i j}(x, D u),
$$

where $\mathcal{L}$ is defined by (2.2). Then we have for tangential derivatives, 


$$
\left|\mathcal{L}\left(D_{\alpha}(u-\underline{u})\right)\right| \leqslant C\left(1+\sum_{i} F^{i i}\right), \quad \alpha=1, \cdots, n-1, \quad \text { in } \Omega .
$$

We also observe that, on $\partial \Omega$ close to the origin, the tangential derivative satisfies $D_{\alpha}(u-\underline{u})=0$, $\alpha=1,2, \cdots, n-1$, i.e., $\left|D_{\alpha}(u-\underline{u})\right| \leqslant C\left|x^{\prime}\right|^{2}$. This implies

$$
\left|D_{\alpha}(u-\underline{u})\right| \leqslant C|x|^{2}, \quad \text { on } \partial \Omega_{\delta}
$$

where $\Omega_{\delta}=\Omega \cap B_{\delta}(0)$ is a small neighborhood of the origin, $\partial \Omega_{\delta}=\left(\partial \Omega \cap B_{\delta}(0)\right) \cup\left(\Omega \cap \partial B_{\delta}(0)\right)$. We always choose $\delta$ so small that $\partial \Omega \cap B_{\delta}(0) \subset T$.

Let $v=1-\phi$, where $\phi=e^{K(\underline{u}-u)}$ is the barrier function in Remark 2.1, then we have

$$
\mathcal{L} v \leqslant-\epsilon_{1} \sum_{i} F^{i i}+C, \text { in } \Omega, \text { and } v=0 \text {, on } \partial \Omega,
$$

for positive constants $\epsilon_{1}$ and $C$. We consider a function of the form

$$
\psi=v+\mu x_{n}-k x_{n}^{2},
$$

where $\mu$ and $k$ are positive constants to be determined. We then have

$$
\mathcal{L} \psi \leqslant-\frac{\epsilon_{1}}{4}\left(1+\sum_{i} F^{i i}\right), \quad \text { in } \Omega_{\delta}, \quad \text { and } \psi \geqslant 0, \text { on } \partial \Omega_{\delta},
$$

for $k$ sufficiently large and $\mu, \delta$ sufficiently small.

By modification of the function $\psi$, we employ a new barrier function

$$
\tilde{\psi}=a \psi+b|x|^{2}
$$

with positive $a$ and $b$ to be determined. By a direct computation, for $a \gg b \gg 1$, we have

$$
\begin{gathered}
\mathcal{L} \tilde{\psi}=a \mathcal{L} \psi+b \mathcal{L}|x|^{2} \leqslant-\left(\frac{a \epsilon_{1}}{4}-b\right)\left(1+\sum_{i} F^{i i}\right), \\
\left|D_{\alpha}(u-\underline{u})\right|=0 \leqslant \tilde{\psi}, \quad \text { on } \partial \Omega \cap B_{\delta}(0),
\end{gathered}
$$

and

$$
\left|D_{\alpha}(u-\underline{u})\right| \leqslant b \delta^{2} \leqslant a \psi+b \delta^{2}=\tilde{\psi}, \text { on } \Omega \cap \partial B_{\delta}(0) .
$$

Therefore, for $a \gg b \gg 1$ and $\delta \ll 1$, there holds

$$
\begin{aligned}
\left|\mathcal{L} D_{\alpha}(u-\underline{u})\right|+\mathcal{L} \tilde{\psi} & \leqslant 0, \quad \text { in } \Omega_{\delta}, \\
\left|D_{\alpha}(u-\underline{u})\right| & \leqslant \tilde{\psi}, \quad \text { on } \partial \Omega_{\delta} .
\end{aligned}
$$

By the maximum principle, we have

$$
\left|D_{\alpha}(u-\underline{u})\right| \leqslant \tilde{\psi}, \text { in } \Omega_{\delta} .
$$

At the origin, we have $\left|D_{\alpha}(u-\underline{u})(0)\right|=\tilde{\psi}(0)=0$. For $\left|D_{\alpha}(u-\underline{u})\right|$, taking $x^{\prime}=0$, dividing by $x_{n}$ and letting $x_{n} \rightarrow 0$, we get

$$
\left|D_{\alpha n}(u-\underline{u})(0)\right| \leqslant a \psi_{n}(0) \leqslant C .
$$

Thus, we obtain the mixed tangential-normal derivative estimate $\left|D_{\alpha n} u(x)\right| \leqslant C, \alpha=1, \cdots, n-1$, on $T$.

It therefore remains to estimate the double normal derivative $D_{n n} u$ and for this the regularity of the matrix function $A$ is critical. Following the idea in [21] as used in [22], we fix a unit vector $\xi \in \mathbb{R}^{n-1}$ and set, (with respect to our transformed coordinates),

$$
\begin{aligned}
w: & =w[u]=w_{\alpha \beta} \xi_{\alpha} \xi_{\beta} \\
& =\left[D_{\alpha \beta} u-A_{\alpha \beta}(x, D u)\right] \xi_{\alpha} \xi_{\beta} \\
& =\left[D_{\alpha \beta} \varphi-A_{\alpha \beta}\left(x, D^{\prime} \varphi, D_{n} u\right)\right] \xi_{\alpha} \xi_{\beta} \text { on } T
\end{aligned}
$$


with $w>0$ by virtue of the ellipticity of $u$. Here $D^{\prime}=\left(D_{1}, \ldots \ldots D_{n-1}\right)$ denotes the tangential gradient. An estimate from above for $D_{n n} u$ on $T$ will follow from equation (1.1), provided we obtain a positive lower bound for $w$ on $T$. To get this we note that for sufficiently large $K$ the function $\tilde{w}=w+K|x|^{2}$ will take a minimum on $T \times S^{n-1}$ at some point $\bar{x} \in T$, and for some unit vector $\bar{\xi}$. Extending $\varphi$ by defining $\varphi\left(x^{\prime}, x_{n}\right)=\varphi\left(x^{\prime}, 0\right)$ for $x_{n}>0$, it follows from the regularity of $A$, that the corresponding extension of $w$ is a concave function of $D_{n} u$. Consequently from the differentiated equation (3.4), for $\alpha=n$, we have

$$
\mathcal{L} \tilde{w} \leqslant C\left(1+\sum_{i} F^{i i}\right)
$$

Now using the barrier in Remark 2.1 as above, we obtain this time a one-sided estimate $D_{n} w(\bar{x}) \geqslant-C$, that is

$$
D_{n n} u(\bar{x}) D_{p_{n}} A_{\alpha \beta}\left(\bar{x}, D^{\prime} \varphi, D_{n} u\right) \bar{\xi}_{\alpha} \bar{\xi}_{\beta} \leqslant C .
$$

To reach our desired estimate for $D_{n n} u$, we then need to get a positive lower bound for the coefficient in (3.18). By the ellipticity of the subsolution $\underline{u}$, we can fix a positive constant $\delta$ for which

$$
w[\underline{u}] \geqslant \delta, \text { for all } x \in T,|\xi|=1 .
$$

We also have

$$
\begin{aligned}
w[u]-w[\underline{u}] & =-\left[A_{\alpha \beta}\left(\bar{x}, D^{\prime} \varphi, D_{n} u\right)-A_{\alpha \beta}\left(\bar{x}, D^{\prime} \varphi, D_{n} \underline{u}\right)\right] \bar{\xi}_{\alpha} \bar{\xi}_{\beta} \\
& \geqslant-D_{n}(u-\underline{u})(\bar{x}) D_{p_{n}} A_{\alpha \beta}\left(\bar{x}, D^{\prime} \varphi, D_{n} u\right) \bar{\xi}_{\alpha} \bar{\xi}_{\beta},
\end{aligned}
$$

since $D_{n} u>D_{n} \underline{u}$ from the strictness of $\underline{u}$ and again using the regularity of $A$. We also have an upper bound

$$
D_{n}(u-\underline{u}) \leqslant \kappa
$$

for a positive constant $\kappa$.

Combining (3.19), (3.20) and (3.21), we thus obtain for $w[u]<\frac{\delta}{2}$,

$$
D_{p_{n}} A_{\alpha \beta}\left(\bar{x}, D^{\prime} \varphi, D_{n} u\right) \bar{\xi}_{\alpha} \bar{\xi}_{\beta}>\frac{\delta}{2 \kappa} .
$$

Hence, we conclude from (3.18) an estimate $D_{n n} u(\bar{x}) \leqslant C$ for a further constant $C$. Now utilizing equation (1.1) again, we obtain an estimate from below for $w(\bar{x})$ and finally an estimate from above for $D_{n n} u\left(x_{0}\right)$. Since an estimate from below automatically follows from the ellipticity of $u$, we complete the estimation of $D^{2} u$ on $\partial \Omega$ and the proof of Theorem 1.2 .

Remark 3.1. In the above proof, we could have used, in place of (3.16), the function

$$
w[u]=\left\{\operatorname{det}\left[w_{i j}\right]\right\}^{\frac{1}{n-1}}
$$

where indices $i, j=1, \cdots, n-1$, in direct accordance with the technique in [21].

Remark 3.2. In [23], the concept of domain A-convexity is introduced extending that of c-convexity in optimal transportation. In particular for $A$ a given $n \times n$ symmetric matrix function defined on $\Omega \times \mathbb{R}^{n}$ we may define $\Omega$ to be uniformly A-convex, with respect to $u$ if

$$
\left[D_{i} \gamma_{j}+D_{p_{k}} A_{i j}(x, D u) \gamma_{k}\right] \tau_{i} \tau_{j} \geqslant \delta_{0}
$$

for all $x \in \partial \Omega$, unit outer normal $\gamma$ and unit tangent vector $\tau$ and some positive constant $\delta_{0}$. It then follows that $\Omega$ is uniformly A-convex if and only if, for any constant $K>0$, there exists a defining function $\varphi \in C^{2}(\bar{\Omega})$ satisfying $\varphi=0$ on $\partial \Omega$ together with the inequality (2.5) in a neighbourhood of $\partial \Omega$. 
By using $\varphi$ in place of the barrier in Remark 2.1, we conclude an estimate for the Hessian $D^{2} u$ on $\partial \Omega$ for solutions of the Dirichlet problem 1.1 for uniformly A-convex domains, thereby proving Theorem 2.2 in [23]. Note that the concept of A-convexity is also invariant with respect to coordinate changes. Note that the replacement of uniform A-convexity by the existence of a strict subsolution with the same boundary trace for second derivative estimates, as formulated in Theorem 2.1, is also pointed out in 23 .

Remark 3.3. Theorem 1.2, provides bounds for both $D^{2} u$ and the augmented matrix function $w$. By the equation (1.1), we have the positive lower bounds for $w$ in $\bar{\Omega}$. Thus, the uniform ellipticity of the operator $F$, with respect to $u$, follows easily from the positive upper and lower bounds of $w$.

\section{EXISTENCE THEOREM AND SOME APPLICATIONS}

In this section, we complete the proof of the classical solvability result for the Dirichlet problem (1.1) and consider the applications to the optimal transportation and prescribed Jacobian equations.

First we establish the necessary solution bounds and gradient bounds for Theorem 1.3. By the comparison principle, we have $u \geqslant \underline{u}$ in $\Omega$. The subsolution $\underline{u}$ is a lower bound of the solution. To obtain an upper bound, suppose $u$ attains its maximum at $x_{0} \in \bar{\Omega}$, that is $D u\left(x_{0}\right)=0$ and $D^{2} u\left(x_{0}\right) \leqslant 0$. By ellipticity, we then have $A\left(x_{0}, 0\right)<0$, which contradicts the hypothesis that $A(x, 0)$ has a non-negative eigenvalue for each $x \in \Omega$ and hence $u$ must take its maximum on $\partial \Omega$. Thus we have the solution bound

$$
|u| \leqslant K_{0}, \text { in } \bar{\Omega},
$$

where $K_{0}$ depends on $\|\underline{u}\|_{L^{\infty}(\Omega)}$ and $\|\varphi\|_{L^{\infty}(\Omega)}$.

The latter condition is equivalent to constant functions being viscosity supersolutions, as defined in [20], so more generally we could assume that there exists a viscosity supersolution $\bar{u} \geqslant \phi$ on $\partial \Omega$.

We assume a quadratic bound from below for the matrix $A$ to control the gradient of the solution, namely that $A$ satisfy the following structure condition (1.6),

$$
A(x, p) \geqslant-\mu_{0}\left(1+|p|^{2}\right) I .
$$

Consider the function $\psi=e^{\kappa u}|D u|$ for some $\kappa>0$. Suppose $\psi$ attains its maximum at $x_{0} \in \Omega$, namely $D_{i} \psi\left(x_{0}\right)=0$. Hence, at the point $x_{0}$, we have $D_{i} u D_{i} \psi=0$. By calculation, we have

$$
\kappa|D u|^{4}+D_{i j} u D_{i} u D_{j} u=0 .
$$

From the ellipticity condition $D^{2} u-A(x, D u)>0$, we have

$$
\kappa|D u|^{4}+A_{i j}(x, D u) D_{i} u D_{j} u<0 .
$$

By the structure condition (1.6) of the matrix $A$, we have

$$
\kappa|D u|^{4}-\mu_{0}\left(1+|D u|^{2}\right)|D u|^{2}<0 .
$$

Without loss of generality, we may assume $D u\left(x_{0}\right) \geqslant 1$. The left hand side of (4.4) will be nonnegative if we choose the positive constant $\kappa$ sufficiently large. This contradiction leads to the gradient estimate

$$
\sup _{\Omega}|D u| \leqslant C,
$$

where $C$ depends on $\mu_{0},\|u\|_{L^{\infty}(\Omega)}$ and $\sup _{\partial \Omega}|D u|$.

On the boundary, the tangential derivatives of $u$ are given by the boundary condition and the interior normal derivative bound from below is controlled by the subsolution $\underline{u}$. The estimate from above follows 
from condition (1.6) and the ellipticity, which implies $\Delta u>A_{i i}$, (see proof of Theorem 14.1 in [11]). Combining with (4.5), we have

$$
|D u| \leqslant K_{1}, \text { in } \bar{\Omega},
$$

where $K_{1}$ depends on $\|\underline{u}\|_{C^{1}(\Omega)}$ and $\|\varphi\|_{C^{1}(\Omega)}$.

As we shall indicate below, for the special cases of prescribed Jacobian and optimal transportation equations, the above conditions can be relaxed.

To complete the proof of Theorem 1.3, we have from Theorem 1.2 and the $C^{1}$ bounds (4.1) and (4.6), uniform estimates in $C^{2}(\bar{\Omega})$ for classical elliptic solutions of the Dirichlet problems:

$$
\left\{\begin{aligned}
\operatorname{det}\left\{D^{2} u-A(x, D u)\right\} & =t B(x, D u)+(1-t) \operatorname{det}\left\{D^{2} \underline{u}-A(x, D \underline{u})\right\} \text { in } \Omega, \\
u & =\varphi \text { on } \partial \Omega,
\end{aligned}\right.
$$

for $0 \leqslant t \leqslant 1$. Theorem 1.3 then follows from the Evans - Krylov second derivative Hölder estimates of Evans, Krylov and Caffarelli-Nirenberg-Spruck, (see for example [11], Theorems 17.26, 17.26'), and the method of continuity ([1], Theorem 17.8). The uniqueness assertion is immediate from the maximum principle.

Remark 4.1. We remark that the classical solution $u$ belongs to $C^{\infty}(\bar{\Omega})$ if both $A$ and $B$ are $C^{\infty}$ functions on $\bar{\Omega} \times \mathbb{R}^{n}$, as well as $\partial \Omega \in C^{\infty}, \varphi \in C^{\infty}(\partial \Omega)$. Under the stated hypotheses it also follows from the linear theory [11] that the solution $u \in C^{3, \alpha}(\bar{\Omega})$ for all $\alpha<1$. In fact we have the stronger inclusion, $u \in W^{4, p}(\Omega)$ for all $p<\infty$ and this would in fact suffice for the argument in Section 2. Note that we only use $u \in C^{3}(\bar{\Omega})$ in Section 3.

Remark 4.2. As a special case for Monge-Ampère type equation (1.1), the existence results for the classical solutions to the Dirichlet problem for the equation $\operatorname{det}\left(u_{i j}+\sigma_{i j}(x)\right)=\psi(x)$ treated in the strictly convex domain in [1, 2] can be generalized to non-convex domains. Also, the equation with general right hand side treated by Li [15] can be generalized to non-convex domains.

Remark 4.3. For the relationship with equations arising in conformal geometry the reader is referred to [9] where a similar barrier argument for boundary estimates is used.

In the remainder of this paper, we discuss the application to optimal transportation and prescribed Jacobian equations. Optimal transportation problems have received a lot of attention in recent years and for the basic theory, we refer to the the books, 30, 31. Letting $Y$ be a $C^{1}$ mapping from $\Omega \times \mathbb{R} \times \mathbb{R}^{n}$ to $\mathbb{R}^{n}$ and $u$ a function in $C^{2}(\Omega)$, we define a mapping $T=T u: \Omega \rightarrow \mathbb{R}^{n}$ by setting $T u=Y(\cdot, u, D u)$. The corresponding prescribed Jacobian equation may be written the form

$$
|\operatorname{det} D T|=\psi(\cdot, u, D u)
$$

for a given positive function $\psi$ on $\Omega \times \mathbb{R} \times \mathbb{R}^{n}$. If $\operatorname{det} D_{p} Y \neq 0$, we can then write equation (4.8), for elliptic solutions $u$, as an equation of Monge-Ampère type

$$
\operatorname{det}\left[D^{2} u-A(\cdot, u, D u)\right]=B(\cdot, u, D u)
$$

with matrix function $A$ and scalar function $B$ given by

$$
\begin{aligned}
& A(\cdot, u, p)=-Y_{p}^{-1}\left(Y_{x}+Y_{u} \otimes p\right), \\
& B(\cdot, u, p)=\left|\operatorname{det} Y_{p}\right|^{-1} \psi,
\end{aligned}
$$

so that when $Y$ and $\psi$ are independent of $u$, (and $B$ is positive), we obtain a Monge-Ampère type equation of the form (1.1) considered here. Optimal transportation equations are the special cases 
where the mapping $Y$ is generated by a cost function $c$ satisfying conditions (A1) and (A2) as formulated in the introduction. We then have, in accordance with (1.5),

$$
\begin{aligned}
Y_{p}^{-1} & =D_{x, y}^{2} c(\cdot, Y) \\
A(\cdot, p) & =D_{x}^{2} c(\cdot, Y) \\
B(\cdot, p) & =\left|\operatorname{det} D_{x, y}^{2} c\right| \psi>0
\end{aligned}
$$

The natural boundary condition for prescribed Jacobian equations is the prescription of the image $T(\Omega)$. The existence of classical solutions is treated in [24], which extends the optimal transportation case in [28]. However the classical Dirichlet problem in small balls has been used for local regularity arguments in [18, 27, 32]. Clearly, the estimates in Theorem 1.1] and 1.2 extend immediately to embrace prescribed Jacobian and optimal transportation equations under regularity of the matrix functions $A$ given by (4.10) and (4.11). For the above solution and gradient estimates some relaxation of our additional hypotheses is possible. First we note that for any vector $\xi \in \mathbb{R}^{n}$, the quantity $T u \cdot \xi$ must assume its maximum and minimum values on the boundary $\partial \Omega$ since $\operatorname{det} D T \neq 0$. In the optimal transportation case this immediately provides a bound for the gradient in term of its boundary trace since $D u=c_{x}(\cdot, T u)$ in $\Omega$. Also in the optimal transportation case, functions of the form $\bar{u}=c\left(\cdot, y_{0}\right)$ are solutions of the homogeneous equation so we automatically obtain solution bounds. For an arbitrary domain $\Omega$, a condition of the type (1.6) would still be required for the boundary gradient estimate or more generally the existence of a supersolution $\bar{u}$ satisfying $\bar{u}=\varphi$ on $\partial \Omega$. However if the solution is $c$-convex in $\Omega$, that is at each $x_{0} \in \Omega$, there exists $y_{0} \in \mathbb{R}^{n}$, such that

$$
u(x) \geqslant u\left(x_{0}\right)+c\left(x, y_{0}\right)-c\left(x_{0}, y_{0}\right)
$$

in $\Omega$, then we have $T u(\Omega) \subset T \underline{u}(\Omega)$ by virtual of the monotonicity property ([18, Lemma 4.3), and the global gradient bound follows immediately. From [27, we know that an elliptic solution will be $c$-convex in $\Omega$ if $\Omega$ is $c$-convex with respect to each $y \in \mathbb{R}^{n}$, that is the images $c_{y}(\cdot, y)(\Omega)$ are convex in $\mathbb{R}^{n}$. Consequently, we have the following existence theorem for optimal transportation equations.

Theorem 4.1. Let $c \in C^{4}\left(\mathbb{R}^{n} \times \mathbb{R}^{n}\right)$ be a cost function satisfying (A1), (A2) with regular matrix function $A$ given by (1.5) and $B \in C^{2}\left(\bar{\Omega} \times \mathbb{R}^{n}\right)$ satisfying inf $B>0$. Suppose there exists a subsolution $\underline{u} \in C^{2}(\bar{\Omega})$ of equation (1.1) in $\Omega$, satisfying $\underline{u}=\varphi$ on $\partial \Omega$. Then if there also exists a supersolution $\bar{u},=\varphi$ on $\partial \Omega$, or if $\Omega$ is c-convex with respect to all $y \in \mathbb{R}^{n}$, there exists a unique classical solution $u \in C^{2}(\bar{\Omega})$ of the Dirichlet problem (1.1) with $u \geqslant \underline{u}$ in $\Omega$.

Remark 4.4. A concept of generalized solution for optimal transportation equations, extending that of Aleksandrov for the standard Monge-Ampère equation, is also introduced in [18] and the Dirichlet problem for generalized solutions is considered in [10]. Without the regularity assumption, this notion can be non-local as shown by Loeper [17] and thus is not really a weak form of the classical notion.

Remark 4.5. These results also extend to the more general Monge-Ampère type equations (4.9) for A and $B$ satisfying

$$
D_{u} A_{i j} \xi_{i} \xi_{j} \geqslant 0, B_{u} \geqslant 0
$$

for all $(x, u, p) \in \Omega \times \mathbb{R} \times \mathbb{R}^{n}$. In this generality, Theorem 4.1 also extends to embrace prescribed Jacobian equations with mappings $Y$ determined by generating functions as in [25]. 


\section{REFERENCES}

[1] Caffarelli, L., Nirenberg, L., Spruck J.: The Dirichlet problem for nonlinear second order elliptic eqations I: MongeAmpère equations. Comm. Pure Appl. Math. 37, 369-402 (1984)

[2] Caffarelli, L., Nirenberg, L., Spruck, J.: Correction to: The Dirichlet problem for nonlinear second order elliptic eqations I: Monge-Ampère equations. Comm. Pure Appl. Math. 40, 659-662 (1987)

[3] Caffarelli, L.: The regularity of mappings with a convex potential. J. Amer. Math. Soc. 5, 99-104 (1992)

[4] Caffarelli, L.: Boundary regularity of maps with convex potentials II. Ann. of Math. 144 , $453-496$ (1996)

[5] Caffarelli, L., Cabré, X.: Fully Nonlinear Elliptic Equations, Colloquium Publications 43, AMS, Providence, RI, (1995)

[6] Delanoë, Ph.: Classical solvability in dimension two of the second boundary value problem associated with the MongeAmpère operator, Ann. Inst. Henri Poincaré, Analyse Non Linéaire, 8, 443-457 (1991)

[7] Guan, B., Spruck, J.: Boundary value problem on $S^{n}$ for surfaces of constant Gauss curvature. Ann. of Math. 138, 601-624 (1993)

[8] Guan, B.: On the existence and regularity of hypersurfaces of prescribed Gauss curvature with boundary. Indiana Univ. Math. J. 44, 221-241 (1995)

[9] Guan, B.: Conformal metrics with prescribed curvature functions on manifolds with boundary. Amer. J. Math. 129, 915-942 (2007)

[10] Gutiérrez, C.E., Nguyen, T.V.: On Monge-Ampère type equations arising in optimal transportation problems. Calc. Var. Partial Differ. Equations. 28, 275-316 (2007)

[11] Gilbarg, D., Trudinger, N.S.: Elliptic Partial Differential Equation of Second Order, Springer-Verlag, Berlin-New York, (2001)

[12] Ivochkina, N.M.: A priori estimates of $\|u\|_{C^{2, \alpha}(\Omega)}$ of convex solutions of the Dirichlet problem for the Monge-Ampère equation. (Russian) Zap. Nauchn. Sem. Leningrad. Otdel. Mat. Inst. Steklov. (LOMI), 96, 69-79 (1980); English transl. J. Soviet Math. 21, 689-697 (1983)

[13] Ivochkina, N.M.: Classical solvability of the Dirichlet problem for the Monge-Ampère equation. (Russian) Zap. Nauchn. Sem. Leningrad. Otdel. Mat. Inst. Steklov. (LOMI), 131, 72-79 (1983); English transl. J. Soviet Math. 30, 2287-2292 (1985)

[14] Krylov, N.V.: Boundedly inhomogeneous elliptic and parabolic equations in a domain. (Russian) Izv. Akad. Nauk SSSR, 47, 75-108 (1983); English transl. Math. USSR-Izv. 22, 67-98 (1984)

[15] Li Y.Y.: Some existence results of fully nonlinear elliptic equations of Monge-Ampère type. Comm. Pure Appl. Math. 43, 233-271 (1990)

[16] Liu, J., Trudinger, N.S.: On Pogorelov estimates for Monge-Ampère type equation. Discrete Contin. Dyn. Syst. Series A. 28, 1121-1135 (2010)

[17] Loeper, G.: On the regularity of solutions of optimal transportation problems, Acta Math. 202, 241-283 (2009)

[18] Ma, X.-N., Trudinger N.S., Wang, X.-J.: Regularity of potential functions of the optimal transportation problem. Arch. Rat. Mech. Anal. 177, 151-183 (2005)

[19] Schulz, F.: Regularity theory for quasilinear elliptic systems and Monge-Ampère equations in two dimensions. Lecture Notes in Math. 1445 (1990)

[20] Trudinger, N.S.: The Dirichlet problem for the prescribed curvature equations. Arch. Rational Mech. Anal. 111, 153-179 (1990)

[21] Trudinger, N.S.: On the Dirichlet problem for Hessian equations, Acta Math. 175, 151-164 (1995) 
[22] Trudinger, N.S.: Lectures on nonlinear elliptic equations of second order. Lectures in Mathematical Sciences 9, Univ. Tokyo, (1995)

[23] Trudinger, N.S.: Recent developments in elliptic partial differential equations of Monge-Ampère type. ICM. Madrid, 3, 291-302 (2006)

[24] Trudinger, N.S.: On the prescribed Jacobian equation, Proc. Intl. Conf. for the 25th Anniversary of Viscosity Solutions, Gakuto Intl. Series, Math. Sci. Appl. 20, 243-255 (2008)

[25] Trudinger, N.S.: On the local theory of prescribed Jacobian equations. Preprint (2012)

[26] Trudinger, N.S., Wang, X.-J.: The Monge-Ampère equation and its geometric applications. Handbook of geometric analysis. No. 1, Adv. Lect. Math. (ALM), 7, 467-524, Int. Press, Somerville, MA, (2008)

[27] Trudinger, N.S., Wang, X.-J.: On strict convexity and continuous differentiability of potential functions in optimal transportation. Arch. Rational Mech. Anal. 192, 403-418 (2009)

[28] Trudinger, N.S., Wang, X.-J.: On the second boundary value problem for Monge-Ampère type equations and optimal transportation. Ann. Scuola Norm. Sup. Pisa Cl. Sci. VIII, 143-174 (2009)

[29] Urbas, J.: On the second boundary value problem for equations of Monge-Ampère type. J. Reine angew. Math. 487, 115-124 (1997)

[30] Villani, C.: Topics in Mass Transportation, Graduate Studies in Mathematics, American Mathematical Society, Providence, Rhode Island (2003)

[31] Villani, C: Optimal Transport - Old and new, Grundlehren, Springer, Berlin (2008)

[32] Wang, X.-J.: On the design of a reflector antenna. Inverse problems. 12, 351-375 (1996)

School of Science, Nanjing University of Science \& Technology, Nanjing 210094, P.R.China

E-mail address: jfd2001@163.com

Centre for Mathematics and Its Applications, The Australian National University, Canberra ACt 0200, Australia

E-mail address: Neil.Trudinger@anu.edu.au

School of Science, Nanjing University of Science \& Technology, Nanjing 210094, P.R.China

E-mail address: yangxp@mail.njust.edu.cn 